\title{
Surface tensions and surface potentials of acid solutions
}

Cite as: J. Chem. Phys. 133, 154107 (2010); https://doi.org/10.1063/1.3505314

Submitted: 28 July 2010 . Accepted: 02 October 2010 . Published Online: 19 October 2010

Alexandre P. dos Santos, and Yan Levin

\section{ARTICLES YOU MAY BE INTERESTED IN}

The Surface Tension of Debye-Hückel Electrolytes

The Journal of Chemical Physics 2, 528 (1934); https://doi.org/10.1063/1.1749522

Detecting the undetectable: The role of trace surfactant in the Jones-Ray effect

The Journal of Chemical Physics 149, 194702 (2018); https://doi.org/10.1063/1.5050421

Solvation of the hydronium ion at the water liquid/vapor interface

The Journal of Chemical Physics 119, 6351 (2003); https://doi.org/10.1063/1.1599274

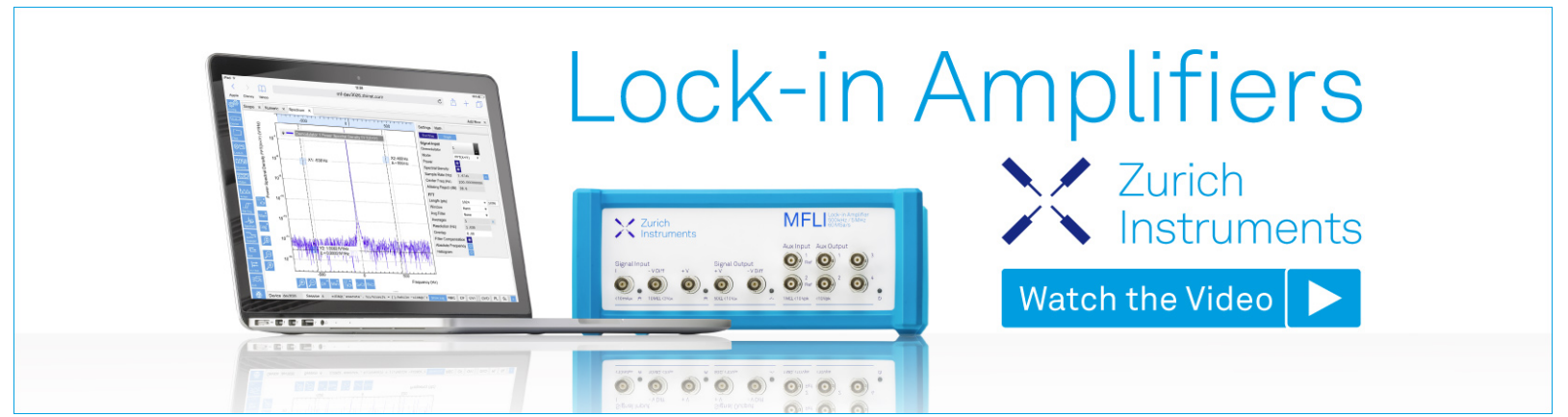

J. Chem. Phys. 133, 154107 (2010); https://doi.org/10.1063/1.3505314 


\title{
Surface tensions and surface potentials of acid solutions
}

\author{
Alexandre P. dos Santos and Yan Levin ${ }^{\text {a) }}$ \\ Instituto de Física, Universidade Federal do Rio Grande do Sul, Caixa Postal 15051, \\ CEP 91501-970 Porto Alegre, Rio Grande do Sul, Brazil
}

(Received 28 July 2010; accepted 2 October 2010; published online 19 October 2010)

\begin{abstract}
A theory is presented which allows us to quantitatively calculate the excess surface tension of acid solutions. The $\mathrm{H}^{+}$, in the form of hydronium ion, is found to be strongly adsorbed to the solution-air interface. To account for the electrostatic potential difference measured experimentally, it is necessary to assume that the hydronium ion is oriented with its hydrogens pointing into the bulk water. The theory is quantitatively accurate for surface tensions and is qualitative for electrostatic potential difference across the air-water interface. (C) 2010 American Institute of Physics.

[doi:10.1063/1.3505314]
\end{abstract}

\section{INTRODUCTION}

Electrolyte solutions are of fundamental interest for a variety of disciplines. Over a hundred years ago, Hofmeister observed a strong dependence of the stability of protein solutions on the specific nature of electrolyte. While some ions tend to stabilize protein solutions, often denaturing them in the process, others destabilize them favoring protein precipitation. A few years after Hofmeister, Heydweiller ${ }^{1}$ observed that salt increases the surface tension of the air-water interface. Furthermore, Heydweiller noticed that the relative effect that ions have on the surface tension follows closely the Hofmeister series, suggesting that the two phenomena are related.

Over the past hundred years, there has been a great effort to understand how ionic specificity influences stability of protein solutions and how it affects the surface tension of the air-water interface. Langmuir ${ }^{2}$ was probably the first to attempt to construct a quantitative theory of surface tensions of electrolyte solutions. Appealing to the Gibbs adsorption isotherm, Langmuir concluded that the excess surface tension of electrolyte solution was a consequence of ionic depletion from the interfacial region. However, no clear explanation for this depletion was provided. A few years after, Wagner ${ }^{3}$ argued that this depletion was the result of interaction between the ions and their electrostatic images across the airwater interface. Onsager and Samaras ${ }^{4}$ simplified Wagner's theory and obtained a limiting law, which they argued was universally valid for all electrolytes at sufficiently small concentration. More recently, Levin and Flores-Mena ${ }^{5}$ used a direct free energy calculation to obtain surface tension of strong electrolytes. To have a quantitative agreement with experiments, these authors stressed the fundamental importance of ionic hydration. Nevertheless, the theory of Levin and Flores-Mena ${ }^{5}$ was not able to predict correctly the surface tensions of all electrolyte solutions. Boström et al. ${ }^{6}$ suggested that the Hofmeister effect and the ionic specificity are a consequence of dispersion forces arising from finite frequency electromagnetic fluctuations. This theory predicted

${ }^{a)}$ Electronic mail: levin@if.ufrgs.br. that weakly polarizable cations should be adsorbed at the air-water interface. This, however, was contradicted by the experimental measurements of the electrostatic potential difference $^{7,8}$ and by the simulations on small water clusters, ${ }^{9-11}$ as well as by the subsequent large scale polarizable force fields simulations, ${ }^{12-15}$ and the photoelectron emission experiments. ${ }^{16-18}$ These experiments and simulations showed that some anions-and not cations-are present at the solution-air interface. ${ }^{9-18}$ To explain this, Levin ${ }^{19}$ extended the traditional Born theory of ionic solvation to account for ionic polarizability. The new theory predicted that highly polarizable anions can actually prefer an interfacial solvation. In a followup work, Levin et al. ${ }^{20,21}$ used this theory to quantitatively calculate the surface tensions and the surface potentials of ten different electrolyte solutions and to reproduce the Lyotropic, Hofmeister, series.

While almost all salts lead to an increase in the air-water surface tension, acids tend to lower it. ${ }^{22,23}$ The only explanation for this is a strong proton adsorption at the water-air interface. ${ }^{12,24-28}$ It is well known that $\mathrm{H}^{+}$ion forms various complexes with water molecules. ${ }^{29-31}$ It has also been suggested that the high surface adsorption of $\mathrm{H}^{+}$is related to the hydronium $\left(\mathrm{H}_{3} \mathrm{O}^{+}\right)$geometry. ${ }^{26}$ This ion has a trigonal pyramidal structure with the hydrogens located at the base of the pyramid. $^{25}$ In this form, hydrogens are good hydrogen-bond donors, while oxygen is a bad hydrogen-bond receptor. ${ }^{26}$ This favors hydronium ion to be preferentially located at the interface, with the hydrogens pointing toward the bulk water and the oxygen pointing into the gas phase. ${ }^{25}$ Explicit solvation energy calculations confirm this picture. ${ }^{32}$

The sign of electrostatic surface potential difference is related to the relative population of cations and anions at the interface. Because of the high adsorption of hydronium ions, one would naturally expect that the electrostatic potential difference across the air-water interface for acid solutions should be positive. The experiments, however, show that the surface potential difference for acids has the same sign as for halide salts, i.e., is predominantly negative. ${ }^{7,23}$ Frumkin $^{7}$ suggested that this apparently strange behavior might be a consequence of the incomplete dissociation of acid molecules. A different explanation was advanced by Randles, ${ }^{33}$ who ar- 
gued that the presence of hydroniums at the interface leads to a preferential orientation of water molecules, resulting in a dipole layer with a negative electrostatic potential difference across it. This conclusion is in agreement with the theory proposed in the present paper, as well as with the recent molecular dynamics simulations. ${ }^{25}$

In this paper, we present a theory that allows us to quantitatively calculate the surface tensions of acid solutions using only one adjustable parameter related to the strength of the hydronium adsorption to the interface. Predictions of the theory are compared with the experimental measurements. The theory is then used to estimate the electrostatic potential difference across the water-air interface for various acid solutions.

\section{MODEL AND THEORY}

We consider an acid solution in a form of a drop of radius $R$, where $r=R$ is the position of the Gibbs dividing surface (GDS). ${ }^{20,34}$ The water and air will be modeled as uniform dielectrics of permittivities $\epsilon_{w}=80$ and $\epsilon_{o}=1$, respectively. The surface tension can be obtained by integrating the Gibbs adsorption isotherm equation,

$$
\mathrm{d} \gamma=-\Gamma_{+} \mathrm{d} \mu_{+}-\Gamma_{-} \mathrm{d} \mu_{-},
$$

where $\mu_{ \pm}=k_{B} T \ln \left(c_{b} \Lambda_{ \pm}^{3}\right)$ are the chemical potentials and $\Lambda_{ \pm}$ are the de Broglie thermal wavelengths. In this equation, the + sign corresponds to the hydronium ion, and the - sign to the anion. The bulk ion concentration is $c_{b}=\rho_{+}(0)=\rho_{-}(0)$, where $\rho_{ \pm}(r)$ are the ionic density profiles. The ion excess per unit area due to existence of the interface is

$$
\Gamma_{ \pm}=\frac{1}{4 \pi R^{2}}\left[N-\frac{4 \pi R^{3}}{3} c_{b}\right]
$$

where $N$ is the total number of acid "molecules." The ionic density profiles $\rho_{ \pm}(r)$, will be calculated using a modified Poisson-Boltzmann (mPB) equation, as discussed later in the paper.
Anions are divided into two categories: kosmotropes and chaotropes. The theory of electrolyte solutions ${ }^{21}$ showed that chaotropes $\mathrm{Br}^{-}, \mathrm{I}^{-}, \mathrm{NO}_{3}^{-}$, and $\mathrm{ClO}_{4}^{-}$loose their hydration sheath near the GDS and are partially adsorbed to the interface. On the other hand, kosmotropes $\mathrm{F}^{-}, \mathrm{Cl}^{-}$, and $\mathrm{SO}_{4}^{2-}$ remain hydrated in the interfacial region and are repelled from the GDS.

To bring an ion of radius $a_{h}$ to the distance $z>a_{h}$ from the GDS requires ${ }^{5}$

$W\left(z ; a_{h}\right)=\frac{q^{2}}{2 \epsilon_{w}} \int_{0}^{\infty} d k e^{-2 s\left(z-a_{h}\right)} \frac{k\left[s \cosh \left(k a_{h}\right)-k \sinh \left(k a_{h}\right)\right]}{s\left[s \cosh \left(k a_{h}\right)+k \sinh \left(k a_{h}\right)\right]}$

of work. In this equation, $s=\sqrt{\left(\kappa^{2}+k^{2}\right)}$ and $\kappa$ $=\sqrt{8 \pi q^{2} c_{b} / \epsilon_{w} k_{B} T}$ is the inverse Debye length. The kosmotropic ions remain strongly hydrated in the interfacial region and encounter a hardcorelike repulsions from the GDS at a distance of one hydrated ionic radius. On the other hand, strongly polarizable chaotropic anions $\left(\mathrm{Br}^{-}, \mathrm{I}^{-}, \mathrm{NO}_{3}^{-}\right.$, and $\mathrm{ClO}_{4}^{-}$) loose their hydration sheath and can move cross the water-air interface. However, to avoid the large electrostatic energy penalty of exposing the charge to a low-dielectric (air) environment, the electronic charge density of a chaotropic anion redistributes itself so as to remain largely hydrated. ${ }^{19}$ The fraction of ionic charge, which remains inside the aqueous environment, $x(z)$, can be calculated by minimization of the polarization energy, ${ }^{19}$

$$
\begin{aligned}
U_{p}(z, x)= & \frac{q^{2}}{2 a_{0} \epsilon_{w}}\left[\frac{\pi x(z)^{2}}{\theta(z)}+\frac{\pi[1-x(z)]^{2} \epsilon_{w}}{[\pi-\theta(z)] \epsilon_{o}}\right] \\
& +\frac{(1-\alpha)}{\alpha \beta}\left[x(z)-\frac{1-\cos [\theta(z)]}{2}\right]^{2} .
\end{aligned}
$$

In the above equation, $\alpha$ is the relative polarizability defined as $\alpha=\gamma_{i} / a_{0}^{3}$, where $\gamma_{i}$ is the ionic polarizability, $a_{0}$ is the unhydrated (bare) radius, and $\theta(z)=\arccos \left[-z / a_{0}\right]$. Performing the minimization, we obtain

$$
x(z)=\left[\frac{\lambda_{B} \pi \epsilon_{w}}{a_{0} \epsilon_{o}[\pi-\theta(z)]}+\frac{(1-\alpha)}{\alpha}[1-\cos [\theta(z)]]\right] /\left[\frac{\lambda_{B} \pi}{a_{0} \theta(z)}+\frac{\lambda_{B} \pi \epsilon_{w}}{a_{0} \epsilon_{o}[\pi-\theta(z)]}+2 \frac{(1-\alpha)}{\alpha}\right],
$$

where $\lambda_{B}=q^{2} / \epsilon_{w} k_{B} T$ is the Bjerrum length.

The force that drives chaotropic ions toward the interface results from water cavitation. To introduce an ion into an aqueous environment requires creating a cavity, which perturbs the hydrogen-bond network of water molecules. For small ions, the free energy cost of forming a cavity is entropic and is proportional to the volume of the void formed. ${ }^{35,36}$ As the ion moves across the GDS, its cavitational free energy decreases. This results in a short-range attractive potential between the anion and the GDS, ${ }^{20}$

$$
U_{\text {cav }}(z)= \begin{cases}\nu a_{0}^{3} & \text { for } z \geq a_{0} \\ \frac{1}{4} \nu a_{0}^{3}\left(\frac{z}{a_{0}}+1\right)^{2}\left(2-\frac{z}{a_{0}}\right) & \text { for }-a_{0}<z<a_{0}\end{cases}
$$

where $\nu \approx 0.3 k_{B} T / \AA^{3}$ is obtained from bulk simulations. ${ }^{37}$ For hard (weakly polarizable ions), the cavitational free energy gain is completely overwhelmed by the electrostatic free energy penalty of moving ionic charge into the low di- 


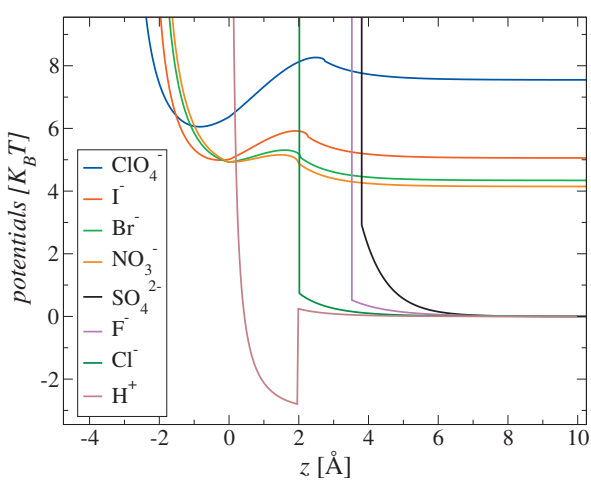

FIG. 1. Potentials for all ions at $1 M$. The GDS is at $z=0 \AA$. The bulk cavitational potential is not considered for kosmotropes since it does not change along the drop.

electric environment. For soft polarizable ions, however, the electrostatic penalty is small, since most of the ionic charges remain inside the aqueous environment. The total potential of a soft anion, therefore, has a minimum in the vicinity of the GDS (see Fig. 1).

The $\mathrm{H}^{+}$ions (protons) do not exist as a separate specie in water. Instead, they form complexes with water molecules, $\mathrm{H}_{3} \mathrm{O}^{+}$and $\mathrm{H}_{2} \mathrm{O}_{5}^{+}$. ${ }^{29-31}$ Because of its favorable geometry (trigonal pyramidal), the hydronium ion $\left(\mathrm{H}_{3} \mathrm{O}^{+}\right)$adsorbs to the water-air interface with a preferential orientation ${ }^{25}$ of oxygen toward the air. We model this attraction by a square well potential with a range of a hydrogen bond, $1.97 \AA$,

$$
U_{\text {hyd }}(z)= \begin{cases}0 & \text { for } z \geq 1.97 \AA \\ -3.05 k_{B} T & \text { for } 0 \leq z<1.97 \AA .\end{cases}
$$

The depth of the potential is then adjusted to obtain the experimentally measured surface tension of $\mathrm{HCl}$. The same potential is then used to calculate the surface tensions of all the other acids. We should stress, however, that one should not attach too much meaning to the specific value of the potential depth. The real proton transfer is a quantum mechanical process; therefore, there is bound to be some arbitrariness in how one models it at a classical level. Here, we have chosen the range of the square well potential to be one hydrogen bond. If one changes this distance, the depth of the potential will have to be modified to obtain an optimal fit of the surface tension of $\mathrm{HCl}$ solution. However, once this is done, the values of the surface tension of the other acids will not be significantly affected. Thus, the strength of $\mathrm{H}^{+}$potential is the only free parameter of the theory. The total potential felt by $\mathrm{H}^{+}$is then $U_{H}(z)=U_{\text {hyd }}(z)+W(z ; 0)$ (see Fig. 1 ).

While the kosmotropic anions feel only the potential $W\left(z ; a_{h}\right)$ and the hardcore repulsion from the GDS, the chaotropic anions are influenced by the total potential ${ }^{20}$

$$
\begin{aligned}
& U_{\mathrm{tot}}(z) \\
& = \begin{cases}W\left(z ; a_{0}\right)+\nu a_{0}^{3}+\frac{q^{2}}{2 \epsilon_{w} a_{0}} & \text { for } z \geq a_{0} \\
W\left(a_{0} ; a_{0}\right) z / a_{0}+U_{p}(z)+U_{\mathrm{cav}}(z) & \text { for } 0<z<a_{0} \\
U_{p}(z)+U_{\mathrm{cav}}(z) & \text { for }-a_{0}<z \leq 0 .\end{cases}
\end{aligned}
$$

In Fig. 1, we plot the potentials felt by various ions at $1 M$
TABLE I. Ion classification into chaotropes (c) and kosmotropes (k). Effective radii (hydrated or partially hydrated) for kosmotropes and (bare) for chaotropes, for which we have also included the polarizabilities from Ref. 38. For kosmotropic ions the polarizability is irrelevant and is not included in the Table.

\begin{tabular}{lccc}
\hline \hline Ions & chao/kosmo & $\begin{array}{c}\text { Radius } \\
(\AA)\end{array}$ & $\begin{array}{c}\text { Polarizability } \\
\left(\AA^{3}\right)\end{array}$ \\
\hline $\mathrm{F}^{-}$ & $\mathrm{k}$ & 3.54 & $*$ \\
$\mathrm{Cl}^{-}$ & $\mathrm{k}$ & 2 & $*$ \\
$\mathrm{Br}^{-}$ & $\mathrm{c}$ & 2.05 & 5.07 \\
$\mathrm{I}^{-}$ & $\mathrm{c}$ & 2.26 & 7.4 \\
$\mathrm{NO}_{3}^{-}$ & $\mathrm{c}$ & 1.98 & 4.48 \\
$\mathrm{ClO}_{4}^{-}$ & $\mathrm{c}$ & 2.83 & 5.45 \\
$\mathrm{SO}_{4}^{2-}$ & $\mathrm{k}$ & 3.79 & $*$ \\
\hline \hline
\end{tabular}

concentration, as a function of the distance from the GDS.

The ionic density profiles can now be obtained by integrating the $\mathrm{mPB}$ equation

$$
\begin{aligned}
& \nabla^{2} \phi(r)=-\frac{4 \pi q}{\epsilon_{w}}\left[\rho_{+}(r)-\rho_{-}(r)\right], \\
& \rho_{+}(r)=\frac{N e^{-\beta q \phi(r)-\beta U_{H}(z)}}{\int_{0}^{R} 4 \pi r^{2} d r e^{-\beta q \phi(r)-\beta U_{H}(z)},} \\
& \rho_{-}^{\text {chao }}(r)=\frac{N e^{\beta q \phi(r)-\beta U_{\text {tot }}(r)}}{\int_{0}^{R+a_{0}} 4 \pi r^{2} d r e^{\beta q \phi(r)-\beta U_{\text {tot }}(r)}}, \\
& \rho_{-}^{\text {kos }}(r)=\frac{N \Theta\left(R-a_{h}-r\right) e^{\beta q \phi(r)-\beta W\left(z ; a_{h}\right)}}{\int_{0}^{R-a_{h} 4 \pi r^{2} d r e^{\beta q \phi(r)-\beta W\left(z ; a_{h}\right)}},}
\end{aligned}
$$

where $\Theta$ is the Heaviside step function, $\rho_{-}^{\text {chao }}(r)$ is the density profile for chaotropic anions, and $\rho_{-}^{\mathrm{kos}}(r)$ for kosmotropic ones.

Once the ionic density profiles are calculated, the surface tensions can be obtained by integrating the Gibbs adsorption isotherm [Eq. (1)]. The ionic radii and polarizabilities are the same as were used in our previous work on surface tension of electrolyte solutions. ${ }^{20,21}$ In Table I, we summarize these data.

The depth of the potential $U_{\text {hyd }}(z)[\mathrm{Eq}$. (7)] is adjusted to fit the $\mathrm{HCl}$ experimental data ${ }^{22}$ (see Fig. 2), this is the only adjustable parameter of the theory. We find that a square well

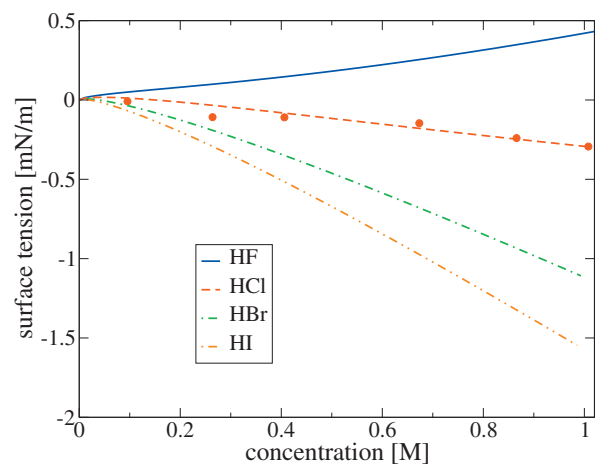

FIG. 2. Surface tensions for $\mathrm{HF}, \mathrm{HCl}, \mathrm{HBr}$, and $\mathrm{HI}$. The symbols are the experimental data for $\mathrm{HCl}$ (Ref. 22), and the lines are the results of the present theory. 


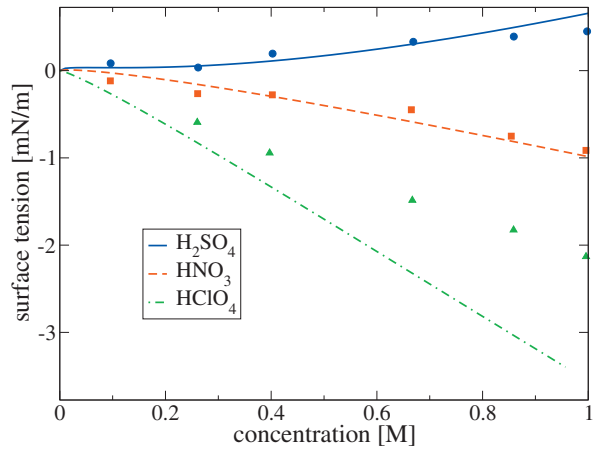

FIG. 3. Surface tensions for $\mathrm{H}_{2} \mathrm{SO}_{4}, \mathrm{HNO}_{3}$, and $\mathrm{HClO}_{4}$. The symbols are the experimental data (Ref. 22) and the lines are the results of the present theory.

potential of depth $-3.05 k_{B} T$ results in an excellent fit of the experimental data for $\mathrm{HCl}$ in the range of concentrations from $0 M$ to $1 M$. The excess surface tension of all other acids is then calculated using the same potential $U_{\text {hyd }}(z)$. The predictions for the surface tensions of $\mathrm{HF}, \mathrm{HBr}$, and $\mathrm{HI}$ are plotted in Fig. 2. Unfortunately, we have no experimental data to compare for these halogen acids. For $\mathrm{H}_{2} \mathrm{SO}_{4}$ and $\mathrm{HNO}_{3}$ (see Fig. 3), we find a good agreement between the theory and experiment. For $\mathrm{HClO}_{4}$, the theory overestimates the surface tension. This is similar to what was found for sodium perchlorate salt. ${ }^{21}$ The difficulty is that $\mathrm{ClO}_{4}^{-}$is a large weakly hydrated ion. Since the cavitational energy grows with the cube of ionic radius, a small error in radius leads to a large error in the surface tension.

Finally, we use the theory to calculate the electrostatic potential difference across the solution-air interface. The surface potential difference, $\Delta \chi=\phi\left(R+a_{0}\right)-\phi(0)$, predicted by the present theory has a wrong sign compared to the Frumkin experimental measurements-positive instead of negative. ${ }^{7,23}$ Positive sign reflects a strong adsorption of hydronium ions to the GDS. The simple dielectric continuum theory presented here, however, does not account for the structure of the interfacial water layer. Since the hydronium ion at the GDS has a preferential orientation with the hydrogens pointing toward the bulk, the presence of many such ions will result in a dipole layer. Note that in the absence of hydroniums, the water dipoles predominantly point along the interface. ${ }^{39}$ The hydronium layer produces an electric field $E=4 \pi p N_{h} / \epsilon_{o} d A$, where $N_{h}$ is the number of hydroniums at the interface, $p$ is the water dipole moment, $d$ is the dipole length, and $A$ is the interfacial area. If we suppose that all the hydroniums are perfectly aligned, the potential difference across the dipolar layer will be $\Delta \chi_{w}=-4 \pi p \Gamma_{+} / \epsilon_{o}$. Using the dipole moment of a water molecule, $p=1.85 \mathrm{D}$, we obtain the dipole layer contribution to the overall electrostatic potential difference. Adding this to $\Delta \chi$, we obtain the total electrostatic surface potential difference across the solutionair interface. In Table II, we list the surface potentials of various acids at $1 M$ concentration. Clearly, these values are an exaggeration of the total electrostatic potential difference across the interface, since at finite temperature there will not be a perfect alignment of interfacial hydronium ions. Nevertheless, the theory should provide us an order of magnitude estimate of the electrostatic potential difference. In fact, for
TABLE II. Surface potential differences for various acids at $1 M$ concentration. Contributions from electrolyte and aligned water dipoles.

\begin{tabular}{lcc}
\hline \hline Acids & $\begin{array}{c}\text { Calculated } \\
(\mathrm{mV})\end{array}$ & $\begin{array}{c}\text { Frumkin }^{\mathrm{a}} \\
(\mathrm{mV})\end{array}$ \\
\hline $\mathrm{HF}$ & 85.5 & -71 \\
$\mathrm{HCl}$ & 1.24 & -23 \\
$\mathrm{HBr}$ & -95 & -34 \\
$\mathrm{HI}$ & -144.8 & -61 \\
$\mathrm{HNO}_{3}$ & -84.4 & -48 \\
$\mathrm{HClO}_{4}$ & -412 & -82 \\
\hline \hline
\end{tabular}

${ }^{\mathrm{a}}$ Reference 7 .

most acids we find a reasonable agreement between the predictions of the theory and the Frumkin experimental measurements. ${ }^{7}$ A noticeable exception is the HF. The experimental potential for hydrogen fluoride measured by Frumkin is negative, while we find a large positive value. The Frumkin value for HF is clearly outside the general trend for halogen acids. In his classical review of electrolyte solutions, Randles ${ }^{33}$ did not mention the Frumkin result for $\mathrm{HF}$ acid, while discussing his other measurements. We can only suppose that Randles also did not have a complete confidence in this particular value. Experimental measurements of excess surface potentials are very difficult. This is probably the reason why the Frumkin measurements of surface potentials of acids have not been repeated in over 90 years.

\section{CONCLUSIONS}

In this paper, we have developed a theory for surface tensions of acid solutions. The hydronium adsorption to the interface was modeled by a square well potential, the depth of which is the only adjustable parameter of the theory. The agreement between the theory and the experiments is very reasonable for different acid solutions at concentrations from $0 M$ to $1 M$. In order to account for the experimental values of the excess electrostatic surface potential, we must require a preferential orientation of hydronium ion at the interface, with the hydrogens pointing into the bulk. With this assumption, we get a qualitative agreement with the experimental measurements of the excess electrostatic potentials of various acid solutions. At the moment, this is the only theory that can account (quantitatively) for the surface tensions and (qualitatively) for the surface potentials of acid solutions.

\section{ACKNOWLEDGMENTS}

This work was partially supported by the CNPq, INCT-FCx, and by the US-AFOSR under Grant No. FA9550-09-1-0283.

\footnotetext{
${ }^{1}$ A. Heydweiller, Ann. Phys. 33, 145 (1910).

${ }^{2}$ I. Langmuir, J. Am. Chem. Soc. 39, 1848 (1917).

${ }^{3}$ C. Wagner, Phys. Z. 25, 474 (1924).

${ }^{4}$ L. Onsager and N. N. T. Samaras, J. Chem. Phys. 2, 528 (1934).

${ }^{5}$ Y. Levin and J. E. Flores-Mena, Europhys. Lett. 56, 187 (2001).

${ }^{6}$ M. Boström, D. R. M. Williams, and B. W. Ninham, Langmuir 17, 4475 (2001).

${ }^{7}$ A. Frumkin, Z. Phys. Chem. 109, 34 (1924).

${ }^{8}$ N. L. Jarvis and M. A. Scheiman, J. Phys. Chem. 72, 74 (1968).

${ }^{9}$ L. Perera and M. L. Berkowitz, J. Chem. Phys. 95, 1954 (1991).
} 
${ }^{10}$ L. X. Dang and D. E. Smith, J. Chem. Phys. 99, 6950 (1993).

${ }^{11}$ S. J. Stuart and B. J. Berne, J. Phys. Chem. A 103, 10300 (1999).

${ }^{12}$ P. Jungwirth and D. J. Tobias, Chem. Rev. 106, 1259 (2006).

${ }^{13}$ P. Jungwirth and D. J. Tobias, J. Phys. Chem. B 106, 6361 (2002).

${ }^{14}$ D. Horinek, A. Herz, L. Vrbka, F. Sedlmeier, S. I. Mamatkulov, and R. R. Netz, Chem. Phys. Lett. 479, 173 (2009).

${ }^{15}$ M. A. Brown, R. D'Auria, I. F. W. Kuo, M. J. Krisch, D. E. Starr, H. Bluhm, D. J. Tobias, and J. C. Hemminger, Phys. Chem. Chem. Phys. 10, 4778 (2008).

${ }^{16}$ G. Markovich, S. Pollack, R. Giniger, and O. Cheshnovski, J. Chem. Phys. 95, 9416 (1991).

${ }^{17}$ S. Ghosal, J. Hemminger, H. Bluhm, B. Mun, E. L. D. Hebenstreit, G. Ketteler, D. F. Ogletree, F. G. Requejo, and M. Salmeron, Science 307, 563 (2005).

${ }^{18}$ B. Garrett, Science 303, 1146 (2004).

${ }^{19}$ Y. Levin, Phys. Rev. Lett. 102, 147803 (2009).

${ }^{20}$ Y. Levin, A. P. dos Santos, and A. Diehl, Phys. Rev. Lett. 103, 257802 (2009).

${ }^{21}$ A. P. dos Santos, A. Diehl, and Y. Levin, Langmuir 26, 10778 (2010).

${ }^{22}$ P. K. Weissenborn and R. J. Pugh, J. Colloid Interface Sci. 184, 550 (1996).

${ }^{23}$ J. E. B. Randles and D. J. Schiffrin, Trans. Faraday Soc. 62, 2403 (1966).

${ }^{24}$ L. M. Levering, M. R. Sierra-Hernández, and H. C. Allen, J. Phys. Chem. C 111, 8814 (2007).
${ }^{25}$ M. Mucha, T. Frigato, L. M. Levering, H. C. Allen, D. J. Tobias, L. X. Dang, and P. Jungwirth, J. Phys. Chem. B 109, 7617 (2005).

${ }^{26}$ M. K. Petersen, S. S. Iyengar, T. J. F. Day, and G. A. Voth, J. Phys. Chem. B 108, 14804 (2004).

${ }^{27}$ P. Petersen and R. J. Saykally, J. Phys. Chem. B 109, 7976 (2005)

${ }^{28}$ S. S. Iyengar, T. J. F. Day, and G. A. Voth, Int. J. Mass. Spectrom. 241, 197 (2005).

${ }^{29}$ M. Eigen, Angew. Chem., Int. Ed. Engl. 3, 1 (1964).

${ }^{30}$ G. Zundel, Adv. Chem. Phys. 111, 1 (2000).

${ }^{31}$ D. Marx, M. E. Tuckerman, J. Hutter, and M. Parrinello, Nature (London) 397, 601 (1999).

${ }^{32}$ L. X. Dang, J. Chem. Phys. 119, 6351 (2003).

${ }^{33}$ J. E. B. Randles, Advances in Electrochemistry and Electrochemical Engineering (Interscience, New York, 1963), Vol. 3.

${ }^{34}$ C. H. Ho, H. K. Tsao, and Y. J. Sheng, J. Chem. Phys. 119, 2369 (2003).

${ }^{35}$ K. Lum, D. Chandler, and J. D. Weeks, J. Phys. Chem. B 103, 4570 (1999).

${ }^{36}$ D. Chandler, Nature (London) 437, 640 (2005).

${ }^{37}$ S. Rajamani, T. M. Truskett, and S. Garde, Proc. Natl. Acad. Sci. U.S.A. 102, 9475 (2005)

${ }^{38}$ N. C. Pyper, C. G. Pike, and P. P. Edwards, Mol. Phys. 76, 353 (1992).

${ }^{39}$ S. M. Kathmann, I. F. W. Kuo, and C. J. Mundy, J. Am. Chem. Soc. 130, 16556 (2008). 